\title{
Chapter 30 \\ Quality Characteristics of Soft Kernel Durum; A New Cereal Crop
}

\author{
Craig F. Morris and E. Patrick Fuerst
}

\begin{abstract}
Production of crops is in part limited by consumer demand and utilization. In this regard, world production of durum wheat (Triticum turgidum subsp. durum) is limited by its culinary uses. The leading constraint is its very hard kernels. Puroindolines, which act to soften the endosperm, are completely lacking in durum. Currently, durum grain is milled on highly specialized mills which produce as their primary objective coarse semolina. Morris and co-workers (2011) described the development of soft kernel durum wheat. The soft kernel trait (Hardness locus) was introgressed into Langdon via Phlb, and crossed to the Italian durum cv. Svevo (producing 'Soft Svevo'). Soft Svevo behaves much like a soft hexaploid wheat with somewhat lower break flour yield, higher water absorption, and smaller cookie diameter. Pilot scale spaghetti manufacture indicated that hydration levels could be reduced to 26-27\% for soft durum, as opposed to about $32 \%$ for commercial semolina. Cooking trials indicated equal-or-better texture, cooking loss and tolerance. Soft Svevo flour performed well in a range of baked goods. These studies demonstrate the stable transfer of the Puroindoline genes from T. aestivum to T. turgidum subsp. durum. As such, the processing and utilization of durum was dramatically altered. The creation of soft durum therefore increases the potential for wheat production under marginal cropping conditions, while establishing a new wheat class with expanded and novel uses.
\end{abstract}

Keywords Durum wheat $\bullet$ Hardness $\bullet$ Kernel texture $\bullet$ Pasta $\bullet$ Puroindoline

\footnotetext{
C.F. Morris $(\square)$

USDA-ARS Western Wheat Quality Lab, E-202 Food Quality Building,

Washington State University, Pullman, WA 99164-6394, USA

e-mail: morrisc@wsu.edu

E.P. Fuerst

Department of Crop and Soil Sciences, Washington State University,

Pullman, WA 99161, USA
} 
Production of specific crops is in part limited by consumer demand and utilization. In this regard, world production of durum wheat (Triticum turgidum subsp. durum (Desf. [Husn.]) is limited by its culinary uses, which are primarily pasta and couscous. Why are the culinary uses of durum wheat more restricted than bread wheat (T. aestivum L.)? Two main genetic systems are responsible: very hard kernels and moderate gluten strength/extensibility. These grain quality traits have influenced the utilization of durum wheat since its domestication, and these traits have defined cultural and social aspects of food in different societies.

Kernel hardness (texture) in wheat is primarily conditioned by the Hardness locus on 5DS which is comprised of Puroindoline $a$ and Puroindoline $b$ (Morris 2002; Morris and Bhave 2008). Puroindolines, which act to soften the endosperm, are completely lacking in durum. Soft wheat has a fully "functional" haplotype, Pina-Dla/Pinb-D1b. Hard hexaploid wheats result from loss-of-function mutations in either Pina or Pinb. To assess kernel texture phenotype, one of the most common methods is the Single Kernel Characterization System (SKCS) (Perten Instruments). The SKCS crushes individual kernels and thus provides data on a "population" of kernels. The mean and standard deviation are diagnostic with regard to interpreting the texture and homogeneity of a particular grain lot. Common results from the SKCS would be $\sim 25$ for soft wheat, $\sim 65$ for hard, and $\sim 85$ for durum.

Currently, durum grain is milled on highly specialized "durum only" mills that can accommodate the very hard kernel texture. Further, these mills have as their primary objective the production of coarse semolina with minimal bran contamination. Some 'flour' is produced, but tends to have high levels of starch damage. Attempts to reduce the particle size of semolina raises starch damage to unacceptable levels. Semolina has about 4-5\% starch damage and a particle size 3-4 times larger than flour. These larger particles require longer hydration times, whereas smaller particles with more starch damage have a higher water requirement. Nonuniform particle size distribution results in uneven hydration and pasta defects. In theory, having a durum wheat with soft kernels would reduce its culinary constraints, and thereby expand its use, demand and production. Since durum can better compete with bread wheat on more marginal lands, soft durum could enhance global food security.

Morris et al. (2011) described in detail the development of soft kernel durum wheat. The soft kernel trait (Hardness locus) originated from Chinese Spring and was introgressed via $P h l b$ homoeologous recombination. Initially, 14 independent recombination lines were isolated in the durum cv. Langdon and assigned a series of numbers, 674-688. Of these, 10 were crossed to the Italian durum cv. Svevo. Soft kernel progeny were obtained from five. A progeny plant from the 674 cross was selected and used in developing a $\mathrm{BC}_{3}$-soft kernel derivative of Svevo. The soft derivative is termed 'Soft Svevo', and has been deposited in the American Type Culture Collection as Accession No. PTA-10087.

Soft Svevo was grown in 2010 at Bozeman, MT, USA under irrigation. Soft Svevo behaves much like a soft hexaploid wheat with somewhat lower break flour yield, higher water absorption, and smaller cookie diameter. Grain protein was $14.2 \%$, with an SKCS hardness of 23.2, compared to typical durum values of $>80$. 
Modified Quadrumat milling produced a break flour yield of $40.5 \%$ and a straightgrade (SG) yield of $68.7 \%$. SG flour protein was $12.0 \%$ with a $0.52 \%$ ash content. Mixograph water absorption was $59.7 \%$. Sugar-snap cookie diameter was $8.97 \mathrm{~cm}$, and sponge cake volume was $980 \mathrm{cc}$. Polyphenol oxidase (PPO) (L-DOPA substrate) was 0.124 AU. Flour SDS sedimentation volume was $11.9 \mathrm{~mL} / \mathrm{g}$. Solvent Retention Capacities (SRC) were Carbonate SRC 74.8 \%, Lactic Acid SRC 97.8 \%, Water SRC 59.1\% and Sucrose SRC $94.1 \%$. For comparison, similar data for cv. Xerpha soft white winter wheat grown at a number of Washington State locations were, break flour yield averaged $44.1 \%$ and SG flour yield was $67.0 \%$. SG flour protein was $8.80 \%$ with a $0.37 \%$ ash content. Mixograph water absorption was $57.0 \%$. Sugar-snap cookie diameter was $9.28 \mathrm{~cm}$. Flour SDS sedimentation volume was $8.5 \mathrm{~mL} / \mathrm{g}$. Carbonate SRC was $69.5 \%$, Lactic Acid SRC $87.3 \%$, Water SRC $56.0 \%$ and Sucrose SRC 79.8\%. Soft Svevo was grown in 2012 at three different locations and management systems in Washington State. Resulting grain proteins ranged from $12.0 \%$ to $15.0 \%$, SKCS hardness ranged from 12.4 to 20.9. Kernel weights ranged from 34.5 to $39.3 \mathrm{mg}$. PPO levels were $0.128-0.140 \mathrm{AU}$.

The Langdon recombination lines were also crossed to the Italian durum cv. Creso and to the Canadian durum cv. Kyle. In both cases, progeny with soft kernels were obtained, consistent with the presence of the Hardness locus and expression of the soft, wild-type Puroindolines from Chinese Spring. Soft Svevo was used as a parent to transfer the soft kernel trait to the adapted high-yielding durum cultivars Alzada, Havasu and Strongfield. Initial and backcross progeny segregated in an expected 1:2:1 ratio for soft kernel indicating stable and predictable inheritance. Recently we have obtained $\mathrm{F}_{2}$ progeny kernels from crosses between Soft Svevo and eleven of the CIMMYT International Durum Yield Nursery (IDYN) entries. All show normal segregation of $F_{2}$ kernels in the spike (see Morris and Beecher 2012).

Pilot scale spaghetti manufacture indicated that hydration levels could be reduced to $26-27 \%$ for soft durum, as opposed to about $32 \%$ for commercial semolina -a reduction of some 15 or more per cent. Less water added means less water to remove with expected significant energy savings. Cooking trials indicated equal-or-better texture, and cooking loss and tolerance.

Soft Svevo flour was used in various baked goods: baguette and batard hearth breads, pan bread, muffins, refrigerated doughs, croissants, and pizza crust. Results were generally very promising. All products showed the creamy yellow color of the durum endosperm carotenoids. Informal taste panelists commented on a very appealing flavor, appearance and texture. More detailed, controlled studies are certainly in order.

These studies demonstrate the stable transfer of the Puroindoline genes and Hardness locus from T. aestivum to T. turgidum subsp. durum. As such, the processing and utilization of durum were dramatically altered. Milling can be performed on "standard" wheat mills. As opposed to coarse semolina, a low starch damage, fine particle size flour can be produced. Flour quality for the first soft durum, 'Soft Svevo' appears to be intermediate between hard and soft hexaploid varieties. Further breeding, selection and testing will be needed to resolve end-use quality in greater detail. However, pilot-scale pasta trials have indicated excellent spaghetti quality of 
soft durum. A number of baked goods, traditionally made from hexaploid wheat flour, have been successfully made. Durums can also be freely crossed with wild emmer wheat, providing direct access to an enormous gene pool of disease resistance and stress tolerance traits. The creation of soft durum therefore increases the potential for wheat production under marginal cropping conditions, while establishing a new wheat class with expanded and novel uses.

Open Access This chapter is distributed under the terms of the Creative Commons Attribution Noncommercial License, which permits any noncommercial use, distribution, and reproduction in any medium, provided the original author(s) and source are credited.

\section{References}

Morris CF (2002) Puroindolines: the molecular genetic basis of wheat grain hardness. Plant Mol Biol 48:633-647

Morris CF, Beecher BS (2012) The distal portion of the short arm of wheat (Triticum aestivum L.) chromosome 5D controls endosperm vitreosity and grain hardness. Theor Appl Genet 125:247-254

Morris CF, Bhave M (2008) Reconciliation of D-genome puroindoline allele designations with current DNA sequence data. J Cereal Sci 48:277-287

Morris CF, Simeone MC, King GE, Lafiandra D (2011) Transfer of soft kernel texture from Triticum aestivum to durum wheat, Triticum turgidum ssp. durum. Crop Sci 51:114-122 